\title{
Platinum nitride with fluorite structure
}

\author{
R. Yu, X. F. Zhang \\ Materials Sciences Division, \\ Lawrence Berkeley National Laboratory, \\ Berkeley, CA94720 \\ February 7, 2005
}

\begin{abstract}
The mechanical stability of platinum nitride has been studied using first-principles calculations. By calculating the single-crystal elastic constants, we show that platinum nitride can be stabilized in the fluorite structure, in which the nitrogen atoms occupy all the tetrahedral interstitial sites of the metal lattice. The stability is attributed to the pseudogap effect from analysis of the electronic structure.
\end{abstract}


Transition metal nitrides are of great interest in both fundamental science and technical applications [1-8]. Most of the early transition metal nitrides are well known hard materials or superconductors. For example, TiN is a widely used hard coating material; NbN has a superconducting critial temperature of $17.3 \mathrm{~K}$ [1]. TiN is also widely used as the diffusion barrier in electronic industry. In contrast, not much success has been achieved in exploring the late transition metal nitrides, especially for platinum group and noble metals [9-10]. Recently, a significant progress in this direction has been made: Platinum nitride, the first binary metal nitride in platinum group, was synthesized under high pressures and temperatures and recovered at ambient conditions [11].

In the present work, we study the mechanical stability of platinum nitride and found that it can be stabilized indeed, although in a crystal structure different from that given in Ref. 11. By calculating the single-crystal elastic constants, we show that platinum nitride is unstable with the zinc-blende structure given in Ref. 11. As shown in Fig. 1a, the N atoms occupy only half of the tetrahedral interstitial sites of the Pt lattice in the zinc-blende structure (PtN). Filling the open structure with another four $\mathrm{N}$ atoms at the remaining tetrahedral sites gives the fluorite structured nitride $\left(\mathrm{PtN}_{2}\right.$, Fig. 1b), which is shown to be mechanically stable. Good consistency between the calculated properties of $\mathrm{PtN}_{2}$ and the experimental results [11] is obtained. 
The electronic structure of $\mathrm{PtN}_{2}$ is given, which shows that the stability of $\mathrm{PtN}_{2}$ can be attributed to the pseudogap effect.

The full-potential linearized augmented plane waves (LAPW) method [12-13] was employed in this study. This method is one of the most accurate schemes in solving the Kohn-Sham equations in density-functional theory [14]. Augmented plane wave plus local orbitals $(\mathrm{APW}+\mathrm{lo})$ [15] were used for valence states, and LAPW was used for the other states, with the RK$\max$ of 8.0. A fully relativistic calculation was performed for core states, whereas the valence states were treated in a scalar relativistic scheme. The total and partial densities of states (DOS) were obtained using a modified tetrahedron method of Blöchl et al. [16] The total Brillouin zones were sampled with $5000 \mathrm{k}$ points. Both the local density approximation (LDA) [17] and the generalized gradient approximation (GGA) [18] exchange correlation functional were employed in the present calculations. Since it is known that the LDA usually underestimates the lattice constants and overestimates the elastic constants, whereas the GGA overestimates the lattice constants and underestimates the elastic constants, we used the arithmetic average of the LDA and the GGA values as the theoretical estimates.

In our calculations, the lattice constants $(a)$ and the bulk moduli $(B)$ were evaluated from the Birch-Murnaghan fit to the total energies as a function of the unit cell volume. The calculation results for $\mathrm{PtN}$ and $\mathrm{PtN}_{2}$ are 
listed in Table 1. The calculations for elemental Pt were also performed for comparison. For Pt, the calculated average values $(a=3.928 \AA, B=279$ GPa) match very well the experimental ones $(a=3.924 \AA[19], B=276$ GPa [20]). Both PtN and $\mathrm{PtN}_{2}$ have lattice constants sufficiently close to the experimental value. As expected, filling four more $\mathrm{N}$ atoms at the tetrahedral sites does not result in a considerable expansion of the unit cell: the calculated lattice constant of $\mathrm{PtN}_{2}$ is only $3.7 \%$ larger than that of $\mathrm{PtN}$. The experimental bulk modulus [11] is significantly larger than the calculated values for $\mathrm{PtN}$ and $\mathrm{PtN}_{2}$. The reason will be discussed later in this Letter.

The mechanical stability of a crystal means that the strain energy must be positive. For a cubic crystal, it implies the following restrictions [21]:

$$
c_{44}>0, c_{11}>\left|c_{12}\right|, \text { and } c_{11}+2 c_{12}>0
$$

where $c_{11}, c_{12}$, and $c_{44}$ are the elastic stiffness constants. In the present work, the elastic stiffness constants were obtained by fitting the total energies of the strained crystals to the third-order polynomial of the strains. Since there are three independent elastic constants for a cubic phase [21], three types of strain, i.e., the volume change, and volume-conserved tetragonal and rhombohedral shear strains, were applied to the optimized structures to calculate the elastic constants.

The total energy as a function of the tetragonal distortion for PtN and 
$\mathrm{PtN}_{2}$ are shown in Figs. 2a and 2b, respectively. The polynomial fit to Fig. 2a gives the shear modulus of $c^{\prime}=\left(c_{11}-c_{12}\right) / 2=-17 \mathrm{GPa}$ for PtN. It means that the zinc-blende PtN is not mechanically stable; the cubic lattice would distort spontaneously to a tetragonal lattice to lower the energy. Fig. $2 \mathrm{~b}$ shows that the fluorite structured $\mathrm{PtN}_{2}$ has positive moduli with the tetragonal distortion and in fact to all the distortions as demonstrated in our calculations; therefore the structure is mechanically stable. The elastic stiffness constants $c_{i j}$, polycrystalline shear modulus $G$, Young's modulus $E$, and Poisson's ratio $v$ were calculated and the results are listed in Table 2. The polycrystalline shear modulus of $G=127 \mathrm{GPa}$, calculated from the single-crystal elastic constants based on the Voigt-Reuss-Hill averaging scheme [22], is about two times the value for the elemental Pt (61 GPa) [20]. From the correlation between the hardness and the shear modulus [23], it is expected that platinum nitride is much harder than Pt. Considering that the density of platinum nitride is only half of that of platinum, the enhancement in hardness is quite remarkable.

We also calculated the X-ray diffraction patterns (not shown here) of $\mathrm{PtN}$ and $\mathrm{PtN}_{2}$ and found no noticeable difference. This was in expectation because the two structures have the same Pt lattice, and the scattering from these heavy metal atoms dominates the X-ray diffraction intensities.

The stoichiometric $\mathrm{PtN}_{2}$ contains 67 at\% N. However, the measured N 
composition was about 50 at\% [11]. Although large errors are generally inevitable in quantitative analysis of light elements by electron microprobe, a nitrogen deficiency may exist indeed in the synthesized phase, like in many anion-deficient fluorite structured fast-ion conductors [24], such as $\mathrm{CeO}_{2}$ and $\mathrm{ZrO}_{2}$. The experimentally determined lattice constant, which is $2.2 \%$ smaller than the calculated value, may be attributed to existence of the nitrogen vacancies. The existence of the vacancies also explains the difference between the measured and the calculated bulk moduli. As seen in Table 1, the measured bulk modulus [11] is about $28 \%$ greater than the calculated value for $\mathrm{PtN}_{2}$. Experimentally, the bulk modulus was derived from the pressure increase with the volume contraction [11]. Since nitrogen was used as the pressure medium, nitrogen atoms could be pressed into the nitrogen-deficient lattice under high pressures and compensate the volume contraction, resulting in an increased bulk modulus. Such a process also implies fast nitrogen diffusion in $\mathrm{PtN}_{2}$, just as the high oxygen mobility in the fast-ion conductors $\mathrm{CeO}_{2}$ and $\mathrm{ZrO}_{2}$.

The electronic structure of $\mathrm{PtN}_{2}$ at zero pressure was calculated. Shown in Fig. 3 is the total density of states. No energy gap is seen, indicating a metallic nature of platinum nitride. There is a deep minimum in DOS at the Fermi level, giving the DOS at the Fermi level $\left(N\left(E_{F}\right)\right)$ of only 0.35 states/eV unit cell. The low $N\left(E_{F}\right)$ indicates that platinum nitride is a poor 
metal, in agreement with the experimental observations [11]. The DOS distribution shown in Fig. 3 manifests a typical stability effect by the formation of a pseudogap [25], where the decrease of total DOS at the Fermi level is accompanied by shifting the occupied bonding states to lower energies and the unoccupied antibonding states to higher energies. The states between -18 $\mathrm{eV}$ and $-13 \mathrm{eV}$ are mainly composed of $\mathrm{N}(2 s)$ states. The states above $-8 \mathrm{eV}$ are mainly composed of $\mathrm{Pt}(5 d)$ states $\left(t_{2 g}\right.$ and $\left.e_{g}\right)$ and $\mathrm{N}(2 \mathrm{p})$ states. Due to the stronger $\operatorname{Pt}\left(t_{2 g}\right)-\mathrm{N}(2 p)$ interactions, the $t_{2 g}$ band has a larger dispersion than that of the $e_{g}$ band, with the antibonding part almost empty.

In summary, the stability of platinum nitride has been investigated using first-principles calculations. It was found that platinum nitride can be stabilized in the fluorite structure, in which the nitrogen atoms occupy all the tetrahedral interstitial sites of the metal lattice. It is interesting to know if other transition metal nitrides, as well as carbides, can be stabilized in this structure. The calculations are in progress and the results will be published elsewhere.

This work was partly supported by the Director, Office of Science, Office of Basic Energy Sciences, Materials Sciences Division of the U.S. Department of Energy under Contract No. DE-AC03-76SF0098. 


\section{References}

[1] L.E. Toth, Transition Metal Carbides and Nitrides (Academic Press, New York, 1971).

[2] H. Pierson, Handbook of Refractory Carbides and Nitrides: Properties, Characteristics and Applications (Noyes Publications, Westwood, New Jersey, 1996).

[3] S.-H. Jhi, J. Ihm, S.G. Louie, and M.L. Cohen, Nature 399, 132 (1999).

[4] J. S. Chun, I. Petrov, and J.E. Greene, J. Appl. Phys., 86, 3633 (1999).

[5] H. Al-Brithen and A. R. Smith, Appl. Phys. Lett. 77, 2485 (2000).

[6] A. Zerr, G. Miehe, and R. Boehler, Nature Mater. 2, 185 (2003).

[7] P. Kroll, Phys. Rev. Lett. 90, 125501 (2003).

[8] V. Ranjan, L. Bellaiche, and E. J. Walter, Phys. Rev. Lett. 90, 257602 (2003).

[9] T. Maruyama, and T. Morishita, Appl. Phys. Lett. 69, 890 (1996).

[10] S. Krishnamurthy, M. Montalti, M. G. Wardle, M.J. Shaw, P.R. Briddon, K. Svensson, M.R.C. Hunt, and L. Šiller, Phys. Rev. B 70, 045414 (2004). 
[11] E. Gregoryanz, C. Sanloup, M. Somayazulu, J. Badro, G. Fiquet, H.-K. Mao, and R.J. Hemley, Nature Mater. 3, 294 (2004).

[12] D.J. Singh, Planewaves, Pseudopotentials and the LAPW Method (Kluwer Academic, Boston, 1994).

[13] P. Blaha, K. Schwarz, G.K.H. Madsen, D. Kvasnicka, and J. Luitz, WIEN2k, An Augmented Plane Wave + Local Orbitals Program for Calculating Crystal Properties (Karlheinz Schwarz, Techn. Universität Wien, Austria), 2001. ISBN 3-9501031-1-2.

[14] P. Hohenberg and W. Kohn, Phys. Rev. 136, B864 (1964); W. Kohn and L.J. Sham, Phys. Rev. 140, A1133 (1965).

[15] E. Sjöstedt, L. Nordström, and D.J. Singh, Solid State Commun. 114, $15(2000)$.

[16] P.E. Blöchl, O. Jepsen, and O.K. Andersen, Phys. Rev. B 49, 16223 (1994).

[17] J.P. Perdew and Y. Wang, Phys. Rev. B 45, 13244 (1992).

[18] J.P. Perdew, K. Burke, and M. Ernzerhof, Phys. Rev. Lett. 77, 3865 (1996). 
[19] W.B. Pearson, A Handbook of Lattice Spacings and Structures of Metals and Alloys (Pergamon Press, New York, 1958).

[20] E.A. Brandes, Smithells Metal Reference Book 6th ed. (Butterworth, London, 1983).

[21] J.F. Nye, Physical Properties of Crystals (Oxford University Press, Oxford, 1985).

[22] R. Hill, Proc. Phys. Soc. London 65, 349 (1952).

[23] D.M. Teter, Mater. Res. Soc. Bull. 23, 22 (1998).

[24] J.C. Boivin and G. Mairesse, Chem. Mater. 10, 2870 (1998).

[25] See, e.g., R. Hoffmann, Solids and Surfaces (VCH Publishers, New York, 1988). 


\section{Figure Captions:}

FIG.1. Unit cell schematics of (a) the zinc-blende structure and (b) the fluorite structure. The large and small spheres denote Pt and $\mathrm{N}$ atoms, respectively. The Pt atoms form a face-centered-cubic (FCC) lattice, which has eight tetrahedral interstitial sites.

FIG.2. Total energies of platinum nitrides as a function of the tetragonal distortion. (a) Zinc-blende phase; (b) Fluorite phase. The zinc-blende phase is not stable against the tetragonal distortion.

FIG.3. Calculated total density of states (DOS) of $\mathrm{PtN}_{2}$. The Fermi level is at 0 . 
Table 1: The lattice constants ( $a$ in $\AA$ ) and bulk moduli ( $B$ in GPa) of platinum nitrides and platinum.

\begin{tabular}{|c|c|c|c|c|c|}
\hline & \multicolumn{3}{|c|}{ Platinum nitrides } & \multicolumn{2}{|c|}{$\mathrm{Pt}$} \\
\hline & exp..$^{[11]}$ & PtN & $\mathrm{PtN}_{2}$ & exp. & cal. \\
\hline & & (cal.) & (cal.) & & \\
\hline \multirow[t]{3}{*}{$a$} & 4.803 & 4.736 & 4.912 & $3.924^{[19]}$ & 3.928 \\
\hline & & $4.692^{1}$ & $4.866^{1}$ & & $3.890^{1}$ \\
\hline & & $4.780^{2}$ & $4.958^{2}$ & & $3.967^{2}$ \\
\hline \multirow[t]{3}{*}{$B$} & 372 & 219 & 290 & $276^{[20]}$ & 279 \\
\hline & & $244^{1}$ & $316^{1}$ & & $320^{1}$ \\
\hline & & $194^{2}$ & $264^{2}$ & & $238^{2}$ \\
\hline
\end{tabular}

$\overline{{ }^{1} \mathrm{LDA} ;{ }^{2} \mathrm{GGA}}$ 
Table 2: The single-crystal elastic constants $c_{i j}$, shear modulus $G$, Young's modulus $E$, and Poisson's ratio $v$ of $\mathrm{PtN}_{2}$. All elastic constants are in GPa, except the dimensionless Poisson's ratio.

\begin{tabular}{ccccccc}
\hline \hline & $c_{11}$ & $c_{12}$ & $c_{44}$ & $G$ & $E$ & $v$ \\
\hline LDA & 532 & 208 & 122 & 137 & 359 & 0.32 \\
GGA & 457 & 167 & 99 & 116 & 303 & 0.31 \\
Ave. & 495 & 188 & 111 & 127 & 332 & 0.31 \\
\hline \hline
\end{tabular}




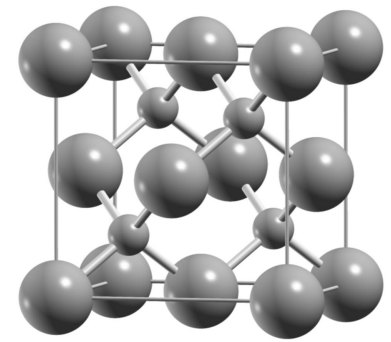

(a)

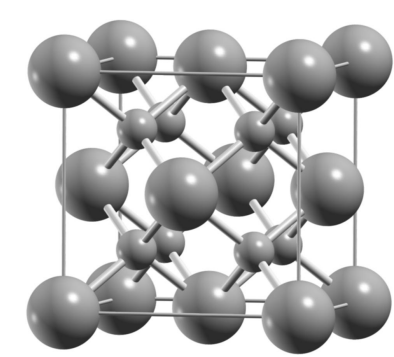

(b) 
(a)

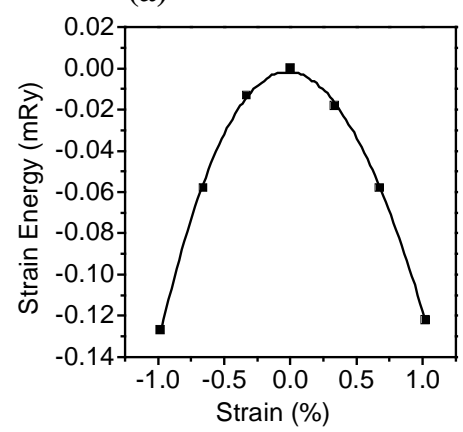

(b)

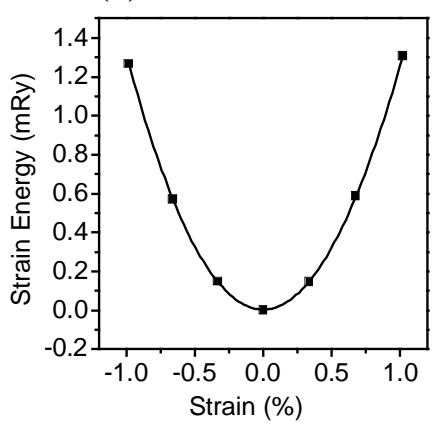




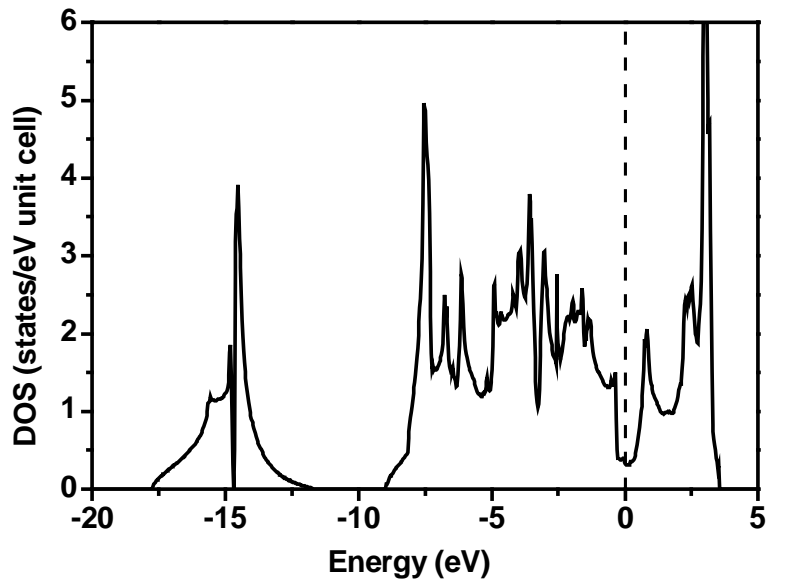

